\title{
Failure Mode and Mechanism in Cermets Under
}

Stress-Wave Loading

K. T. RAMESH, B. ALTMAN, G. RAVICHANDRAN and S. NEMAT-NASSER

Center of Excellence for Advanced Materials, Department of Applied Mechanics and Engineering Sciences, University of California, Mechanics and Engineerng Solla, CA 92093, USA

\section{ABSTRACT}

peries of plate impact recovery experiments on Some preliminare reported. The experimental procedure is boron carbide-aluminum cermets discussed, and the specimen are explored.

\section{KEYWORDS}

Plate impact recovery test; boron carbide-aluminum cerme

\section{INTRODUCTION}

This is a summary of some preliminary results obtained in a series of dynamic plate impact recovery experiments perormed of the University of the Center of Excellence for Advanced Materials (Co these experiments is to California, San Diego (UCSD). One of the alm composites under very understand and quantify the failure modes ive short duration, high-amplitude compressive pulse duration, the avalable energy and unloading with high stress amplimited. Hence, the samples can be overall level of damage recovered and evaluated, us characterization techniques, see relasic measurement of the displacement and Together with direct inter the momentum trap (Barker and Hollenbach, velocity of the back face of the experimental procedure to evaluate damage 1965), this provides a powerful expert failure modes of such brittle bu initiation, damage evolutier, various stress pulses. 


\section{EXPERTMENTS}

The experiments are performed using a gas gun with a $2 \frac{3}{2}$ inch bore, capable of attaining projectile velocities of up to $200 \mathrm{~m} / \mathrm{s}$. The projectile carries a thin flyer plate which impacts the specimen at a predetermined velocity. The flyer plate and the specimen faces are made parallel prior to impact, using an optical allgnment technique; Kunar and clifton (1977). The experiments are fully linstrumerted allowing for careful measurement of the displaceloct an ar face interferometry; see the sketch in Fig. 1.

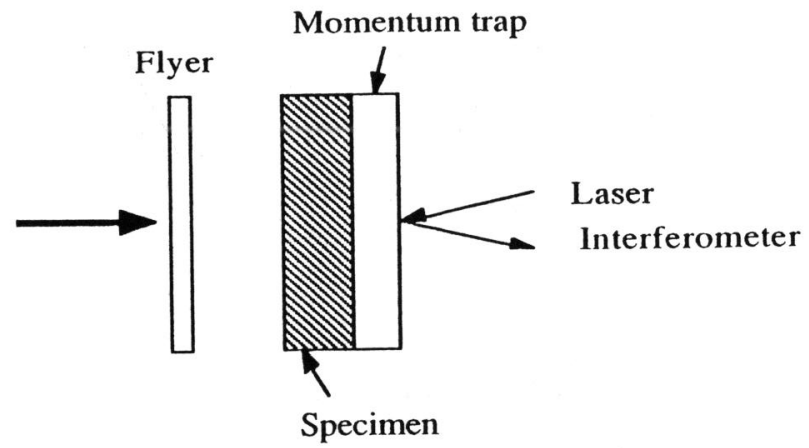

Fig. 1. Plate impact recovery experiment.

The deviation from normal impact, i.e. the tilt, is measured from the instances when the four pins, one at each corner of the specimen, contact the flyer plate. The momentum trap whose impedance matches that of the specimen, captures the pulse and carries away the tensile wave which is reflected from its back face, once this tensile wave reaches the interface between the momentum trap and the specimen. Thus the specimen can be subjected to a compressive pulse with known amplitude and duration, and then the characteristics of this pulse can be measured directly during the experiment. Furthermore, by choice of a suitable material with relatively high yield stress for the nomentum trap, and by limiting the impac velocity, it is assured that no plastic flow occurs in the momentum trap, and therefore, changes in the stress pulse measured at the back face of the momentum trap can be used to assess the nature of the damage that it has produced in the specimen.
A gap can be provided between the momentum trap and the specimen, which then Aroduces a short tensile pulse of a duration corresponding to the time required for the closure of the gap. This pulse travels back as a release pulse and subjects a small region near the impact face of the specimen to tensile loading. By controlling the gap size, we can control the thickness of the region which subsequent to the initial compression loading and unloading, has also experienced tensile loading. A typical x-t diagram for the plate impact recovery test is shown in Fig. 2, including the effect of

\section{t-X DIAGRAM}

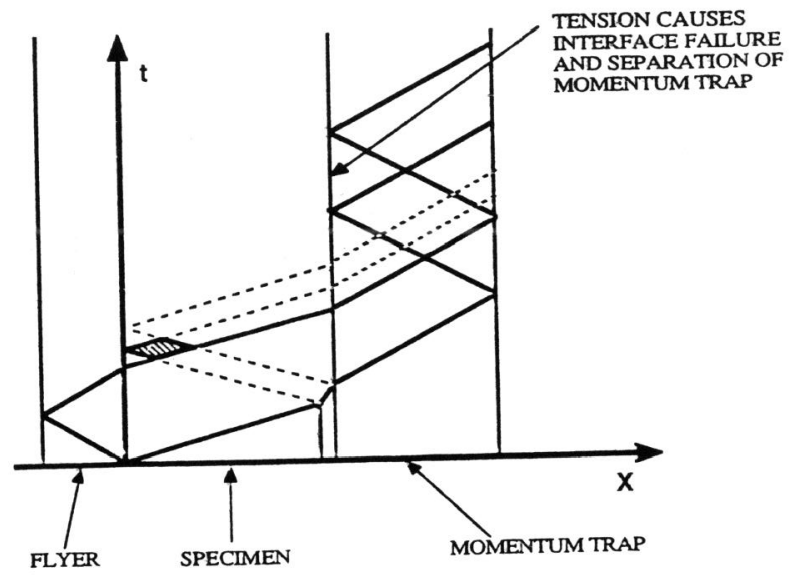

Fig. 2. The $X-t$ diagram corresponding to the plat mpact recovery experiment, including the the specimen and the momentum trap.

the small gap between the momentum trap and the specimen interface. Upon impact, compressive waves travel through the specimen and the flyer plate. When the pulse reaches the gap, it is reflected as a tensile pulse, until the gap is closed, at which time the compression pulse continues to trave into the mon they and are separated from the specimen. The short duration release pulse due to the specimen and can subject a small region of the

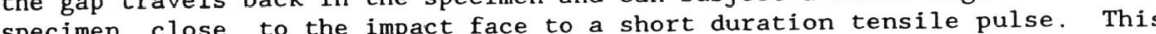
puls reflects back as a compression pulse and, after traveling through the ppecimen behind the main compression pulse, it is transmitted to the momentum trap and is recorded through interferometric measurement. It may provide the means for assessing the damage level in the specimen. 


\section{MATERIALS}

The specimens are made of a class of cermets of 558 volume fraction boron Laboratory. The material is provided by the Lawrence Livermore National Halverson et a material is processed using infiltration National from 0.5 - 2 al. (1986). The boron carbide particles, with sizes rangin aggregate is $\mathrm{m}$, are compacted and chemically treated. The compaing composite is heat th molten 7075 aluminum. The resulting of the composite the Vicker scale. The ul tra speeds are $11.7 \mathrm{~mm} \mu \mathrm{s}$. squares of about 6 me specimens are 25

acoustic plate and the momentum trap are made of a Ti-6Al-4V alloy. The flyer plate is also $25 \mathrm{~mm}$ squarerial loading pulse of about 0.5 ss in elctroplated with a 25 m thick lauration. facilitate the interferometric

\section{RESULTS AND DISCUSSION}

Data corresponding to four tests are summarized in Table 1 , where $U_{0}$ is the Figure 3 shows the $\sigma_{o}$ is the stress amplitude, and $t_{L}$ is the pulse duration. of the momews the resulting particle velocity measured at pulse duration.

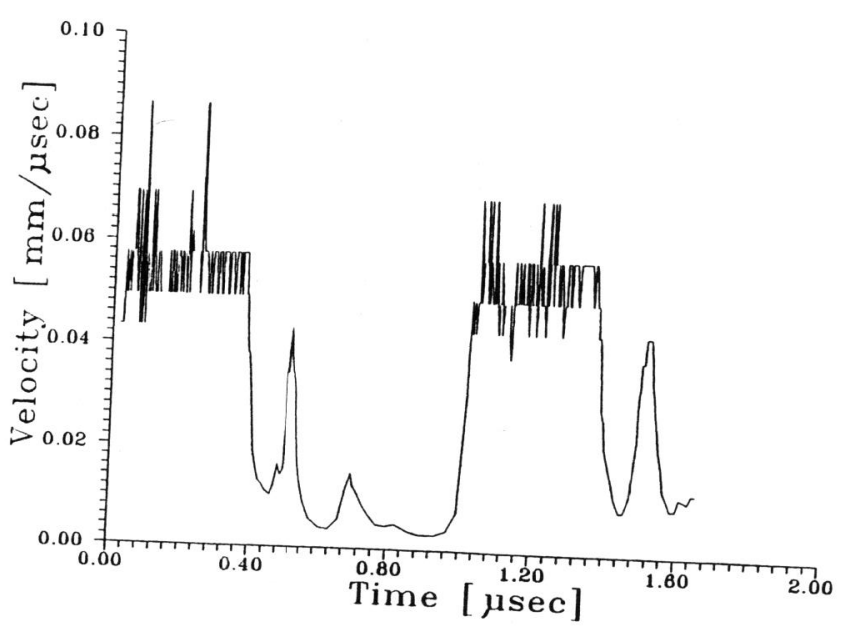
Fig. 3. Measured velocity profile at the back face of
the momentum trap. sequences. These may include: (1) microcracking due to initial compression by the primary pulse; (2) microcracking due to unloading and reloading, as the tensile pulse travels back along the specimen; (3) tensile cracking, as the reflected tensile pulse crosses the primary compression close to the impact face of the specimen; (4) microcracking caused by the unloading of the primary compression pulse; and, finally (5) additional microcracking due to loading and unloading, as part of the short tensile pulse reflects back from the impact face or the fractured region close to the impact face, as a short compression pulse with a reduced amplitude. It is reasonable to expect that most of the energy of the short tensile pulse is actually lost in the process of tensile cracking close to the impact face.

Figures $4 \mathrm{a}, \mathrm{b}$ are micrographs showing the damage in the main part of the specimen (Fig. 4a), and close to the impact face (Fig. 4b). The axial crack axial compression heterogeneous brittle solids; see Horii and Nemat-Nasser $(1985,1986)$.

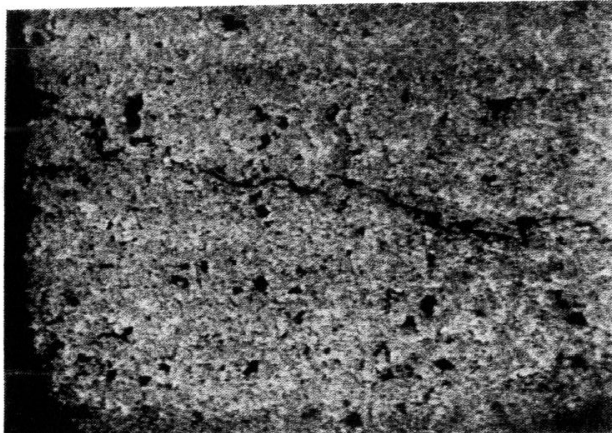

Fig. 4a. Micrograph of recovered specimen; disintegrated boron carbide particles are connected by axial microcracks.

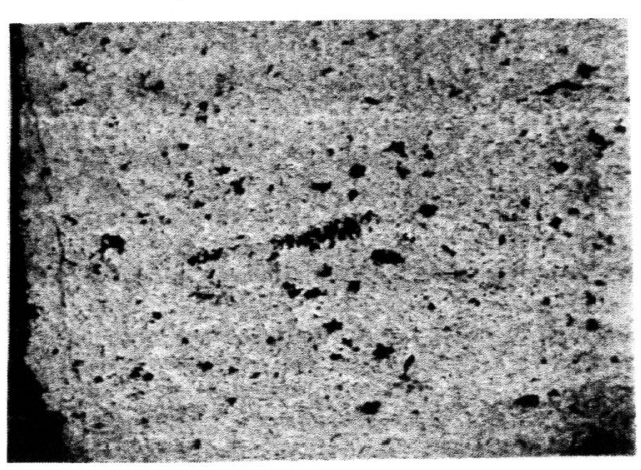

Fig. 4b. Micrograph of recovered specimen; tensile cracks parallel to the impact face, produced by the short tensile pulse which is reflected from the gap bet 
Table 1. Summary of experimental conditions

\begin{tabular}{lccc} 
Experiment & $\begin{array}{l}\text { Uo } \\
\mathrm{m} / \mathrm{s}\end{array}$ & $\begin{array}{l}\sigma_{\circ}^{\circ} \\
\mathrm{MPa}\end{array}$ & $\begin{array}{l}\mathrm{t}_{\mathrm{L}} \\
\mu \mathrm{S}\end{array}$ \\
\hline $\mathrm{KT}-11$ & 45 & 600 & 0.47 \\
\hline $\mathrm{KT}-12$ & 60 & 800 & 0.50 \\
\hline $\mathrm{BA}-14$ & 58 & 770 & 0.44 \\
\hline $\mathrm{BA}-15$ & 71 & 950 & 0.43 \\
\hline
\end{tabular}

The specimen in this

test the measured width of the compressive no visible damage. In this less than $0.44 \mu \mathrm{s}$ which is the value expectis was about $0.4 \mu \mathrm{s}$ which is The compression pulse was followed by a shor $\mu s$ and of considerably smaller amplitude. This pulse of width of about 0.05 the pulse due to and the momentum trap. This tensile pulse propagates onc it the can produce additional pulse, it subjects and cracking

is subjected to the following sequence impact face, most of the sample compression due to the incoming primaryce of loading and unloading: (1) tensile pulse reflected from the gap between the (2) unloading due to the (3) recompression as the tensile pulse moves specimen and the momentum compressive pulse the gap closes; (4) unloading as the tail of the primary reloading, as the es along the specimen and into the momentum trap. from the impact face, or the as a compression puls been formed very the , and finally (6) unloading, momentum trap.

The region close to the impact face of the spe four loading and unloading sequences, but specimen experiences the first the tail of the primary compression but as the short tensile pulse crosses tensile stresses which produce tensile cracks paralujects this region to prack tensile cracks paral to the impact face of

Had the sample remained fully elastic,

reflected tensile pulse would travel , with no damage whatsoever, the interface with the flyer plate and the specimen the sample, would reach the compression pulse with equal amplitude, would, travel be reflected as a the the momentum trap to be measured as a compression pul transmitted to this pulse primary compression pulse. The reduction pulse of an amplitude
It consists of disintegrated zones within the boron carbide that are 列 列 compacted partion and perpendicular to parallel the compressive stress. Since each particle within this region of the specimen undergoes three compressive particis and three unloadings, it is reasonable to expect that compressed aggregates of boron carbide may disintegrate, forming regions containing fragmented boron carbide.

\section{ACKNOWLEDGEMENT}

This work has been supported by the U.S. Army Research Office under Contract No. DAAL-03-86-K-0169 to the University of California, San Diego. The authors thank the Chemistry and Materlals Science Deparom samples.

\section{REFERENGES}

Barker, L. B. and R. E. Hollenbach (1965). Interferometer technique for

measuring the dynamic mechanical properties of materials.
Instr., 36, 1617-1620. Halverson, D. C., A. J. Pyzik, Lawrence Livermore Processing Technical Report, UCRL-93862, 1-9.

Lompression-induced microcrack growt olids: Axial splitting and shear failure. J. Geophys. Res. $90,3105-3125$

ri Splitting faulting and brittle-ductile transition. Phil. Trans. Roy. Soc of London, A-319, 337-374.

Kumar, P and R. J. Clifton (1977). A star-shaped flyer for plate impact Kumar, P. experiments. J. App1. Phys., 48, 4850-4852.

Kumar, $P$ and R.J.Clifton (1979). Dislocation motion and generation in Li single crystals subjected to plate impact. J. App1. Phys., 50, 4747-4762. 